\title{
COUNTERFEITING
}

\section{The fluorescence of fraud}

With the continual advances in printing technology, it is becoming increasingly difficult to discern counterfeit bank notes from genuine US Federal Reserve notes through visual examination. Now, writing in the online journal Optics Express, Thomas Chia and Michael Levene of Yale University report that fluorescence lifetime measurements can reliably spot the difference (Opt. Express 17, 22054-22061; 2009).

There are three common types of counterfeit paper money: digitally copied counterfeits that use a consumergrade colour inkjet or laser printer; traditional counterfeits printed using more sophisticated methods such as a printing press; and bleached paper money made by removing the ink from a lower denomination bill and then reprinting a larger denomination over the ink-less paper.

The paper used in authentic US bank notes is predominately a blend of cotton and linen fibres, and Chia and Levene report that when exposed to a laser beam,

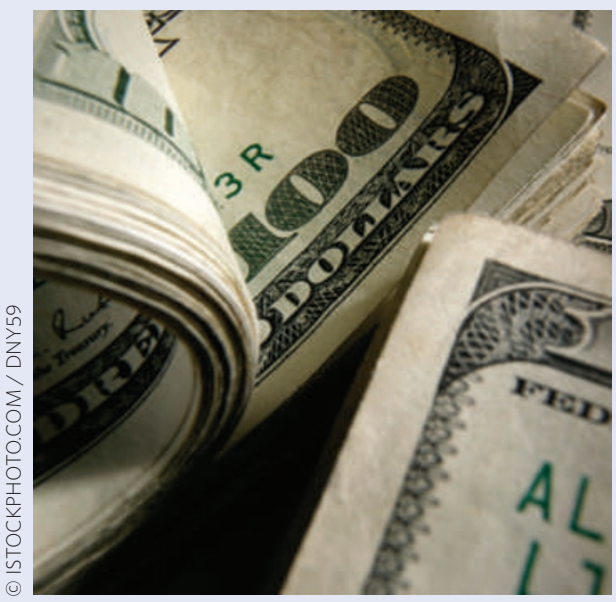

repetition rate, 100 fs pulse duration) Ti:Sapphire laser with emission centred at $735 \mathrm{~nm}$. The optical power at the sample was around $15 \mathrm{~mW}$. A fluorescence lifetime decay curve was produced by raster scanning the laser beam over a $4 \mathrm{~mm}^{2}$ area and capturing the emitted photons for 60 seconds ( 0.8 seconds per frame). The lifetime decay curves were fit to the two-exponential component lifetime model. For genuine US paper money, the average short lifetime component lasted $162 \pm 4.6 \mathrm{ps}$ and the long component lasted $2010 \pm 64$ ps. These values did not change significantly for old notes suffering from wear and tear. In contrast, the long component lifetime of counterfeit notes had a significantly shorter tail, allowing for repeatable and reliable discrimination. It is believed that the technique may be useful to forensic science laboratories for examining very high-quality counterfeits. genuine notes emit fluorescence with a unique time signature.

With assistance from the US government, the Yale University researchers used two-photon microscopy to study a selection of official and fake bank notes. The excitation source was a pulsed $(80 \mathrm{MHz}$

\section{Probing the quantum vacuum}

Researchers are proposing a new experiment that will probe fundamental aspects of the quantum vacuum by searching for highly elusive photon-photon scattering events.

\section{Mattias Marklund}

$\longrightarrow$ uantum mechanics and special relativity have both caused a fundamental shift in our understanding of the world. The marriage between these theories gave birth to quantum electrodynamics (QED), which describes the interaction between light and matter and has been confirmed to exceptional experimental accuracy. Although many decades have passed since Feynman, Schwinger and Tomonaga received the Nobel Prize for their contributions to QED, the theory still gives rise to new discoveries and applications. The latest result, reported in Nature Photonics ${ }^{1}$, comes from Ben King, Antonino Di Piazza and Christoph Keitel at the Max Planck Institute for Nuclear Physics in Heidelberg,
Germany. They describe in detail a new way of looking for exceedingly weak photon-photon scattering events, which are expected to take place in a vacuum exposed to very large laser intensities. The approach relies on searching for a telltale interference pattern similar to that generated by a pair of classical double-slits, although in this case no slits actually exist.

Currently, there is rapid development in high-intensity laser technology for use in both applied and basic research. Applications range from hadron therapy to fusion studies, and fields of intense scientific study include wakefield acceleration, laboratory astrophysics and generation of short-pulse terahertz radiation $^{2,3}$. Moreover, the two large European research infrastructure proposals under development - Extreme Light Infrastructure (ELI) and the High Power Laser Energy Research facility $(\mathrm{HiPER})^{4-7}$ - will make relativistic proton beams and nonlinear quantum vacuum effects accessible.

In the early days of quantum mechanics, the perception of the vacuum as a void empty of structure was understood to be an oversimplified picture. Owing to mass-energy equivalence and Heisenberg's uncertainty relation, the vacuum can be perceived as a bubbling 'soup' of particle pairs that appear and disappear on short timescales. If enough energy is supplied to this vacuum then particle pairs can tunnel from a virtual to a real state. One source of energy for this tunnelling comes in the form of light. Assisting such pair 